\title{
(6) OPEN ACCESS \\ Vitrectomy with or without encircling band for pseudophakic retinal detachment: a multi-centre, three-arm, randomised clinical trial. VIPER Study Report No. 1-design and enrolment
}

\author{
B Mazinani, ${ }^{1}$ S Baumgarten, ${ }^{1}$ P Schiller, ${ }^{2}$ H Agostini, ${ }^{3}$ H Helbig, ${ }^{4}$ E Limburg, ${ }^{5}$ \\ M Hellmich, ${ }^{2} \mathrm{P}$ Walter, ${ }^{1}$ for the VIPER Study Group
}

- Additional material is published online only. To view please visit the journal online (http://dx.doi.org/10.1136/ bjophthalmol-2015-306732).

${ }^{1}$ Department of

Ophthalmology, RWTH Aachen University, Aachen, Germany ${ }^{2}$ Institute of Medical Statistics, Informatics and Epidemiology (IMSIE), University of Cologne, Köln, Germany

${ }^{3}$ Eye Center, Albert-LudwigsUniversity, Freiburg, Germany ${ }^{4}$ Department of

Ophthalmology, University Hospital Regensburg,

Regensburg, Germany ${ }^{5}$ Clinical Trials Center Cologne (ZKS), University of Cologne, Köln, Germany

Correspondence to Dr B Mazinani, Department of Ophthalmology, RWTH Aachen University, Pauwelsstr. 30 Aachen 52074, Germany; bmazinani@gmx.de

Received 9 February 2015 Revised 3 June 2015 Accepted 28 June 2015 Published Online First 21 July 2015
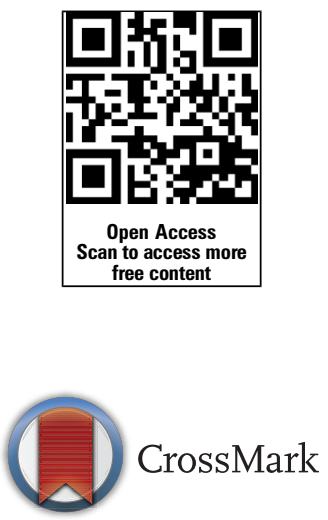

To cite: Mazinani $B$ Baumgarten $S$, Schiller $P$, et al. Br J Ophthalmol 2016:100:405-410.

\section{ABSTRACT}

Purpose Scleral buckling is currently used in addition to vitrectomy for the treatment of pseudophakic retinal detachment (PRD) to better support the vitreous base and better visualisation of the periphery.

Aims The aims of this study are to evaluate (1) whether the combination of $20 \mathrm{G}$ vitrectomy and scleral buckling is superior to $20 \mathrm{G}$ vitrectomy alone (control) (confirmatory), and (2) whether transconjunctival $23 / 25 \mathrm{G}$ vitrectomy is non-inferior to $20 \mathrm{G}$ vitrectomy (both without scleral buckling) regarding operation success (exploratory).

Methods The VIPER (Vitrectomy Plus Encircling Band Vs. Vitrectomy Alone For The Treatment Of Pseudophakic Retinal Detachment) study is an unmasked, multi-centre, three-arm randomised trial. Patients with PRD were eligible, excluding complicated retinal detachment or otherwise severe ophthalmologic impairment. Patients were randomised to one of three interventions: $20 \mathrm{G}$ vitrectomy alone (control C), combination of $20 \mathrm{G}$ vitrectomy and circumferential scleral buckling (experimental treatment E1) or $23 / 25 \mathrm{G}$ vitrectomy alone (experimental treatment E2). The primary endpoint is the absence of any indication for a retina re-attaching procedure during 6 months of follow-up. Secondary endpoints include best corrected visual acuity, retina reattaching procedures, complications and adverse events. Results From June 2011 to August 2013, 257 patients were enrolled in the study. The internet randomisation service assigned 100 patients each to the treatment arms $\mathrm{C}$ and $\mathrm{E} 1$, and 57 patients to treatment $\mathrm{E} 2$. The imbalance is due to the fact that several retinal surgeons did not qualify for performing E2. The random assignment was stratified and balanced (ie, 1:1 or 1:1:1 ratio) by surgeon.

Conclusions The described study represents a methodologically rigorous protocol evaluating the benefits of three different vitrectomy approaches to PRD. The projected results will help to establish their overall efficacy and will permit conclusions regarding their relative value.

Trial registration number DRKS00003158 (German Clinical Trials Register, DRKS).

\section{INTRODUCTION}

\section{Background and rationale}

Vitrectomy has been combined with scleral buckling in the treatment of pseudophakic retinal detachment (PRD) ever since vitrectomies have been used to treat this disorder. Retinal surgeons expect additional buckling procedures to provide enhanced support of the vitreous base and better visualisation of the periphery. On the other hand, the additional scleral buckle implies increased invasivity and operation time, it affects the postoperative refraction and the blood circulation of the eye $\mathrm{e}^{1}$ and may entail specific complications such as buckle migration and infection. The literature is inconclusive regarding the question whether these costs of the additional buckle are associated with increased anatomical or functional success. While some studies reported almost complete success of combined surgery, ${ }^{2}{ }^{3}$ other studies comparing vitrectomy versus vitrectomy with additional buckle found no benefit of the latter. Pournaras and Kapetanios achieved excellent re-attachment rates with both techniques (ie, 100\% vs $92 \%$ at first attempt). ${ }^{4}$ Wickham and coauthors compared vitrectomy alone with vitrectomy and scleral buckling in cases with inferior breaks. In both groups, about half of the patients were pseudophacic: 54 and 53\%, respectively. The primary re-attachment rate was $89 \%$ in the vitrectomy alone group and $73 \%$ in the vitrectomy plus buckle group. ${ }^{5}$ Stangos and coauthors compared vitrectomy alone with vitrectomy plus scleral buckling for PRD. The re-attachment rate was $97 \%$ in the vitrectomy alone group and $92 \%$ in the vitrectomy plus buckle group. ${ }^{6}$ Recently, data on 4179 patients with retinal detachment have been published based on surveys among retinal surgeons. A subgroup analysis showed a slightly higher failure rate for combined surgery versus vitrectomy alone; however, bias due to differential selection of cases (eg, favouring an additional buckle in difficult situations) cannot be ruled out. ${ }^{7}$ All these studies were either retrospective or prospective non-randomised trials. The Scleral buckling versus primary vitrectomy in rhegmatogenous retinal detachment (SPR) study was a prospective randomised multi-centre trial to compare vitrectomy and scleral buckling in the treatment of phakic or pseudophakic patients with retinal detachment of medium complexity. ${ }^{8}$ An ancillary analysis showed that in pseudophakic patients treated with vitrectomy the use of an additional buckle resulted in a significantly lower re-detachment rate of $11.4 \%$ versus $40.9 \%$ in patients who were treated by vitrectomy alone. However, the use of an additional buckle was not randomly assigned but at the discretion of the treating surgeon. In recent years, transconjunctival $23 \mathrm{G}$ or $25 \mathrm{G}$ surgery has been replacing the $20 \mathrm{G}$ approach with exposition of the sclera. Several 
retrospective studies found similar results of the two techniques in the treatment of retinal detachment. ${ }^{9-13}$ Again, evidence from randomised trials is still lacking.

\section{Objectives}

The primary objective of the VIPER (Vitrectomy Plus Encircling Band Vs. Vitrectomy Alone For The Treatment Of Pseudophakic Retinal Detachment) study is to test the superior efficacy of an additional encircling band in addition to a $20 \mathrm{G}$ vitrectomy with gas (treatment codes E1 and C) in the treatment of PRDs (confirmatory). The primary endpoint is the absence of any situation leading to an additional retina re-attaching surgical procedure during the follow-up. The secondary objective is to investigate whether $23 / 25 \mathrm{G}$ transconjunctival vitrectomy with gas (E2) is not inferior to $20 \mathrm{G}$ vitrectomy with gas $(\mathrm{C})$ in the treatment of PRD without encircling band (exploratory).

\section{Trial design}

VIPER is a multi-centre, randomised clinical trial with three parallel treatment arms (see figure 1); however, each comparison (superiority, non-inferiority) is based on two arms only (ie, E1 vs $\mathrm{C}$, and $\mathrm{E} 2$ vs $\mathrm{C}$, respectively). As the study treatments are different surgical procedures, blinding of the trial surgeons is not possible. Given the specific side effects of the additional encircling band such as myopic shift of about $2 \mathrm{D}$ and possible complications such as infection, strabismus, explant intrusion and others, patient blinding is not possible either. The study protocol is available as supplementary file.

\section{PARTICIPANTS AND METHODS}

\section{Study setting}

Patients were enrolled at 14 trial centres specialised on retinal surgery. The participating institutions are university clinics that provide emergency care and treat the majority of regional cases of PRD.

Participating surgeons had to confirm that they had treated at least 100 cases of retinal detachments with primary vitrectomy using a $20 \mathrm{G}$ approach of which at least 20 had to be combined surgery with vitrectomy plus encircling band. Moreover, for the secondary objective regarding $23 / 25 \mathrm{G}$ vitrectomy selected surgeons were required to have performed at least 20 surgical procedures for retinal detachment with $23 / 25 \mathrm{G}$ vitrectomy in addition to the 100 cases with $20 \mathrm{G}$ vitrectomy, and the surgeon had to state that he or she felt safe and comfortable with the transconjunctival technique.

\section{Eligibility criteria}

Pseudophakic patients over 18 years of age were eligible if they suffered from a rhegmatogenous retinal detachment and gave written informed consent. Cataract surgery had to be done at least 3 months before the onset of symptoms of retinal detachment. The main exclusion criteria were manifest uveitis, uncontrolled glaucoma, active retinal vascular disease, malignant intraocular tumours, any history of intraocular surgery other than (distant) cataract surgery, giant retinal tears, proliferative vitreoretinopathy grade $\mathrm{B}$ or $\mathrm{C}$, aphakia and any systemic disorder potentially preventing (i) local/general anaesthesia or (ii) participation in the control examinations.

\section{Interventions}

Control group (C): Patients who were randomly assigned to the control group received a $20 \mathrm{G}$ vitrectomy without encircling band. Surgery was performed with an operating microscope and a wide field viewing system (contact/non-contact). The conjunctiva was opened at the limbus to expose the sclera. Three $20 \mathrm{G}$ sclerotomies were made with a distance of $3-4 \mathrm{~mm}$ to the

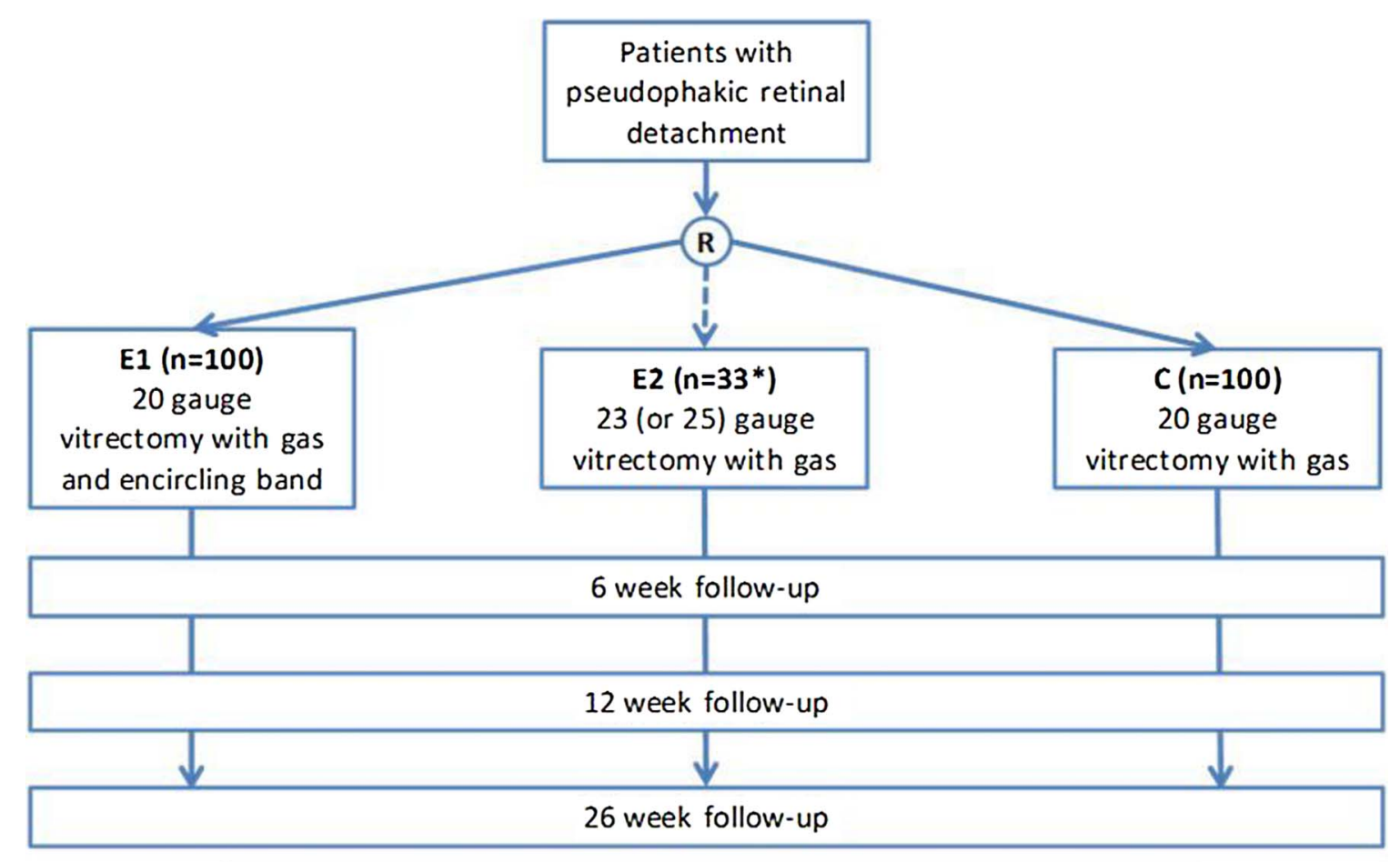

*E2 will only be performed by selected (experienced) study surgeons

Figure 1 Design of the VIPER study. 
limbus. A full vitrectomy was performed. If the vitreous was not fully detached, a complete vitreous detachment was achieved. Heavy liquids were allowed to drain subretinal fluid. After full re-attachment of the retina under heavy liquids or under air, each retinal break was treated with endolaser or transscleral cryopexy. Peripheral high-risk degenerations should be treated as well. A prophylactic circumferential laser treatment was not allowed. The surgery was completed with a gas fill using nonexpandable air/gas mixtures such as SF6, C2F6 or C3F8 and closure of the sclerotomies and the conjunctiva. Ocular pressure had to be monitored at least once within $8 \mathrm{~h}$ after surgery and the day after surgery. If the intraocular pressure exceeded $40 \mathrm{~mm} \mathrm{Hg}$, gas should be released via the pars plana using sterile techniques.

Experimental group 1 (E1): Patients in this group received a $20 \mathrm{G}$ vitrectomy with an encircling band. Surgery started with a circumferential opening of the conjunctiva at the limbus. A $2-4 \mathrm{~mm}$ encircling band was placed underneath the recti muscles and was fixated in all four quadrants. The encircling band was positioned onto the equator of the globe. At the end of surgery, sufficient buckling with no choroidal folds should be visible. The $20 \mathrm{G}$ vitrectomy was performed as described above for the control group (C).

Experimental group 2 (E2): Patients in this group were treated with a $23 \mathrm{G}$ or $25 \mathrm{G}$ vitrectomy without an encircling band. A full vitrectomy was performed using valved or unvalved transconjunctival trocar systems. Trocars were inserted tangentially after displacement of the conjunctiva. If not yet present a full vitreous detachment should be achieved. Endodrainage of the subretinal fluid was achieved with the use of heavy liquids and/ or air. After full re-attachment of the retina, breaks and highrisk degenerations were treated with the endolaser probe or with transconjunctival/transscleral cryopexy. A circumferential prophylactic laser treatment was not allowed. After full fluid-air exchange, the eye was filled with a non-expandable air/gas mixture. Trocars were removed. If the sclerotomies were leaking, they had to be sutured either transconjunctivally or after opening of the conjunctiva.

All groups (C, E1, E2): In none of the groups, the use of silicone oil, internal limiting membrane peeling or triamcinolone was allowed. Medical treatment could be given by the decision of the surgeon and according to institutional standards.

\section{Outcomes}

The primary endpoint was defined as the absence of an indication for any retina re-attaching procedure during the follow-up. Retina re-attaching procedures were additional gas injection, additional vitrectomy or additional buckling procedure. The presence of any such indication was regarded as treatment failure. Generally, functional (eg, visual acuity) or anatomic (as in this study) endpoints are possible to assess treatment success in retinal detachments. In our opinion, visual acuity is not a valid parameter because patients with retinal detachment present in emergency settings during the night or on weekends possibly with a dilated pupil which may affect exact preoperative EDTRS visual acuity testing. In fact almost all studies on retinal detachment use anatomical endpoints. ${ }^{2-7}$ Further, supporting (secondary) endpoints on efficacy and safety are given in table 1 .

After completion of the study follow-up, all documented clinical data (including fundus drawings and photographs) were evaluated by a clinical endpoint committee (SB, BM, PW) regarding the incidence of key study events.

\section{Participant timeline}

Following inclusion of a patient in the study, randomisation and surgery, patients were examined after 6, 12 and 26 weeks (see table 2).

\section{Sample size}

In the SPR study, $11.4 \%(=10 / 88)$ of pseudophakic patients who had received combined primary vitrectomy and scleral buckling suffered from a re-detachment, in contrast to $40.9 \%(=18 / 44)$ of pseudophakic patients who received primary vitrectomy only. ${ }^{8}$ We calculated 82 patients per group $(\mathrm{E} 1, \mathrm{C})$ (software PS V.3.0.43; http://biostat.mc.vanderbilt.edu/PowerSampleSize) for the continuity corrected $\chi^{2}$ test to have $80 \%$ power at two-sided type I error rate of $5 \%$ assuming event proportion of $35 \%$ (E1) and $15 \%(\mathrm{C}) .{ }^{14}$ According to Donner (1984), about 10\% inflation seems adequate to account for the stratification (ie, $91 \approx 82$ /

Table 1 Key secondary endpoints evaluated in the VIPER study

\begin{tabular}{|c|c|c|c|c|}
\hline Endpoint & Measurement variable & Analysis metric & $\begin{array}{l}\text { Method of } \\
\text { aggregation }\end{array}$ & Time \\
\hline Visual acuity & LogMAR visus using ETDRS charts & Change from baseline & Means & At 26 weeks post-surgery \\
\hline Refractive status & Sphere (D), cylinder (D), axis $\left({ }^{\circ} A\right)$ & Change from baseline & Means & At 26 weeks post-surgery \\
\hline Intraocular pressure & Tonometry (mm Hg) & Change from baseline & Means & At 26 weeks post-surgery \\
\hline Retina re-attachment & $\cdots$ & Value (yes/no) & Proportions & $\begin{array}{l}\text { At surgery } \\
\text { At } 26 \text { weeks post-surgery }\end{array}$ \\
\hline Retina-specific procedures & $\ldots$ to achieve a stable retinal attachment & $\begin{array}{l}\text { Value (yes/no) } \\
\text { Count }(>0)\end{array}$ & $\begin{array}{l}\text { Centiles } \\
\text { Proportions }\end{array}$ & Within 26 weeks \\
\hline Proliferative vitreoretinopathy grade C & ... according to Machemer & Value (yes/no) & Proportions & Within 26 weeks \\
\hline Operation time & Time between cut and suture & Value & Means & At surgery \\
\hline Anatomical situation & $\begin{array}{l}\ldots \text { of lids, conjunctiva, cornea, anterior chamber, iris, } \\
\text { intraocular lens or vitreous cavity }\end{array}$ & $\begin{array}{l}\text { Value (yes/no) } \\
\text { Count }(>0)\end{array}$ & $\begin{array}{l}\text { Centiles } \\
\text { Proportions }\end{array}$ & Within 26 weeks \\
\hline Intraoperative complications & ... as iatrogenic breaks, bleeding, sclera perforation & $\begin{array}{l}\text { Value (yes/no) } \\
\text { Count }(>0)\end{array}$ & $\begin{array}{l}\text { Centiles } \\
\text { Proportions }\end{array}$ & At surgery \\
\hline Postoperative complications & $\begin{array}{l}\ldots \text { as macular pucker, macular oedema, ocular } \\
\text { hypertony, diplopia }\end{array}$ & $\begin{array}{l}\text { Value (yes/no) } \\
\text { Count }(>0)\end{array}$ & $\begin{array}{l}\text { Centiles } \\
\text { Proportions }\end{array}$ & Within 26 weeks \\
\hline Enucleation & $\ldots$ & Value (yes/no) & Proportions & Within 26 weeks \\
\hline Death & $\cdots$ & $\begin{array}{l}\text { Time to event } \\
\text { Value (yes/no) }\end{array}$ & $\begin{array}{l}\text { Centiles } \\
\text { Proportions }\end{array}$ & Within 26 weeks \\
\hline
\end{tabular}


Table 2 Visit schedule

\begin{tabular}{|c|c|c|c|c|c|c|}
\hline & $\begin{array}{l}\text { Enrolment } \\
\text { (day }-2 \text { to } 0 \text { ) }\end{array}$ & $\begin{array}{l}\text { Surgery } \\
\text { (day 0) }\end{array}$ & $\begin{array}{l}\text { Week } 6 \\
\text { (W5-7) }\end{array}$ & $\begin{array}{l}\text { Week 12 } \\
\text { (W10-14) }\end{array}$ & $\begin{array}{l}\text { Week 26 } \\
\text { (W23-29) }\end{array}$ & $\begin{array}{l}\text { Additional visit* } \\
\text { (W1-29) }\end{array}$ \\
\hline \multicolumn{7}{|l|}{ Enrolment } \\
\hline Screening for eligibility & $\mathrm{x}$ & & & & & \\
\hline Informed consent & $x$ & & & & & \\
\hline Allocation & $x$ & & & & & \\
\hline \multicolumn{7}{|l|}{ Interventions } \\
\hline Surgery (C, E1, E2) & & $\mathrm{x}$ & & & & \\
\hline \multicolumn{7}{|l|}{ Assessment } \\
\hline Demographic data, medical history & $\mathrm{x}$ & & & & & \\
\hline Best corrected visual acuity (ETDRS) & $x$ & & $x$ & $x$ & $\mathrm{x}$ & $X^{*}$ \\
\hline Refraction & $x$ & & $x$ & $x$ & $x$ & $X^{*}$ \\
\hline Intraocular pressure & $x$ & & $x$ & $x$ & $x$ & $X^{*}$ \\
\hline Indication for retina re-attaching procedure & & $\mathrm{x}$ & $x$ & $x$ & $x$ & $x$ \\
\hline Anatomical findings & $\mathrm{x}$ & & $x$ & $\mathrm{x}$ & $x$ & $X^{*}$ \\
\hline Slit lamp & $x$ & & $\mathrm{x}$ & $x$ & $x$ & $X^{*}$ \\
\hline Funduscopy & $\mathrm{x}$ & & $\mathrm{x}$ & $x$ & $x$ & $X^{*}$ \\
\hline Fundus drawing & $x$ & & $\mathrm{x}$ & $x$ & $x$ & $X^{*}$ \\
\hline Fundus photography & & & & & $x$ & $X^{*}$ \\
\hline Operation time & & $\mathrm{x}$ & & & & \\
\hline Intraoperative complications & & $\mathrm{x}$ & & & & \\
\hline Postoperative complications & & & $x$ & $x$ & $x$ & $X^{*}$ \\
\hline AEs/SAEs & & $\mathrm{x}$ & $\mathrm{x}$ & $x$ & $x$ & $X^{*}$ \\
\hline End of treatment/study & & & & & $\mathrm{x}$ & $X^{*}$ \\
\hline
\end{tabular}

0.9 per group). ${ }^{15}$ Another $10 \%$ was added to account for inevaluable patients (ie, $100 \approx 91 / 0.9$ ). After reaching this target, recruitment to the whole trial was stopped, that is, when, according to expectation, about 33 patients would have been allocated to group E2.

Expecting an event percentage of $35 \%$ for treatment C, we considered a difference smaller than 5 percentage points clinically non-inferior. This corresponds to an OR of about 1.25 which was used as the non-inferiority margin for the comparison of treatments C and E2. Assuming event percentages of $35 \%$ in both arms and a non-inferiority bound of 5 percentage points, 1427 patients per treatment arm are required to reach $80 \%$ power at $2.5 \%$ one-sided type I error rate (calculated with R V.3.0.3, package gsDesign, function nBinomial). Such a big trial seems unfeasible, thus the comparison of $\mathrm{C}$ and $\mathrm{E} 2$ can be explorative only.

The issue of an 'underpowered comparison' was also raised by the local ethics committee. We argued that little evidence of high quality (ie, based on a small number of randomised patients) (i) is better than no evidence at all, at least for the time being, and (ii) may later be incorporated in a meta-analysis of similar cases. This argumentation was approved upon by the local ethics committee. Note also that E2 has already become popular (eg, due to shorter operation time, less postoperative discomfort and logistics) without any evidence from randomised trials.

\section{Allocation}

Patients were consecutively screened and eligible patients were included in the trial. After written informed consent, patients were assigned to one of three treatment groups. In order to achieve comparable intervention groups, patients will be allocated concealed by preoperative randomisation at the day of surgery using a 24/7-internet-service (ALEA, FormsVisions BV, Abcoude, the Netherlands).

Randomisation was stratified by surgeon (permuted blocks of varying length, ie, either two or three occurrences per block). In case of inavailability of the service, patients could be centrally assigned using a prepared sequence of random numbers (ie, by fax, phone or email). The randomisation ratio was either $1: 1: 1$ or $1: 1$ depending on individual experience/training in $23 / 25 \mathrm{G}$ vitrectomy (E2).

We planned to enrol patients at 14 centres with two participating surgeons per centre on average. From a survey among interested surgeons, we estimated that about one-third would be eligible to do E2. Thus, we expected 19 surgeons to enrol for C, E1 and 9 surgeons to enrol for C, E1, E2. Assuming equal enrolment per surgeon (ie, about three per arm), we estimated that overall patients would be assigned to treatment arms in a ratio of $3: 3: 1$ (C:E1:E2).

\section{Methods against bias}

Selection bias is minimised by central $24 / 7$ internet randomisation. Performance bias is minimised by standardisation of trial treatments and including only experienced surgeons. Note that masking of patients or surgeons is not possible. Attrition bias is minimised by provision of excellent care and dedicated follow-up efforts. Detection bias is minimised by using standardised outcome assessment, for example, based on ETDRS visus charts. Note that masking of outcome assessors (ie, the clinical endpoint committee) is generally not possible (eg, due to fundus drawings/photographs).

\section{Data collection and management}

All patient data were remotely entered by clinical centre staff into a validated (ie, US Food And Drug Administration 21 Code 
of Federal Regulations Part 11 compliant) electronic database and checked for plausibility and completeness (central monitoring). Moreover, fundus drawings and 9-field fundus photographs were sent to the coordinating investigator. On-site monitoring could not be performed due to limited funding. Access to the database was restricted to the participating surgeons and monitors. The access of the surgeons was restricted to their own patients. Patient data were pseudonymised.

\section{Statistical methods}

Three trial populations are evaluated: (i) intention-to-treat (ITT) population (all trial subjects enrolled and randomised; analysis as assigned), (ii) per-protocol (PP) population (all trial subjects treated and observed according to protocol) and (iii) the as-treated (AT) population (all trial subjects enrolled and randomised; analysis as treated). The primary analysis of E1 versus C (superiority) is derived from the ITT principle, that is, all patients randomised are analysed as assigned. A missing primary endpoint is considered a treatment failure. Otherwise (secondary) the last observation may be carried forward and/or multiple imputation may be done. For the exploratory comparison of E2 versus $\mathrm{C}$ (non-inferiority), the analyses based on the ITT analysis set and the PP analysis set are considered equally important. ${ }^{16}$ Further details are laid out in the statistical analysis plan (see Supplementary file).

The primary target variable is the number of patients with 'absence of an indication for any retina re-attaching procedure within 26 weeks after surgery'. The treatment comparison E1 versus $\mathrm{C}$ is evaluated for superiority by the Cochran-MantelHaenszel test stratified by surgeon and the corresponding common OR. ${ }^{17}$ Heterogeneity due to surgeon (ie, treatment by surgeon interaction) is tested by the Breslow-Day test. Any significant heterogeneity is attempted to be explained by relevant covariates. For the exploratory comparison of E2 versus C, a non-inferiority margin of 1.25 (OR) is employed. Logistic regression and multiple imputation methods are used for sensitivity analysis.

The secondary endpoints are evaluated by Cochran-MantelHaenszel methods (nominal variables) or linear models (metric variables), respectively, stratified by surgeon. Mixed models for repeated measures, generalised estimating equations and multiple imputations methods are used for sensitivity analysis. Safety data, that is, adverse events and/or complications, are summarised by type, seriousness, intensity, relatedness and the treatment performed. A subgroup analysis is done with respect to sex (the expected proportion of men is $73 \%$ ). No formal interim analysis was planned.

\section{Ethical and legal aspects}

The study was designed and conducted according to the principles of Good Clinical Practice (GCP, ICH E6) and data protection laws. Important protocol modifications can only be made if agreed by the coordinating investigator, the project manager and the statisticians and all authors of the trial protocol. Any changes must be made in writing and must be documented with reasons. They will be signed by all authors of the original trial protocol. Amendments that require approval are submitted to the ethics committee and will not be implemented until approved. Exceptions to this are amendments made to avoid immediate dangers.

The study was prospectively registered in the German Clinical Trials Register under DRKS00003158.

It is planned to publish the trial results, in mutual agreement with the Principal Coordinating Investigator (PCI), in a scientific journal and at German or international congresses. Publication of the results of the trial as a whole is intended. Any publication will take account of the 'Uniform requirements for manuscripts submitted to biomedical journals (International Committee of Medical Journal Editors)'. ${ }^{18}$

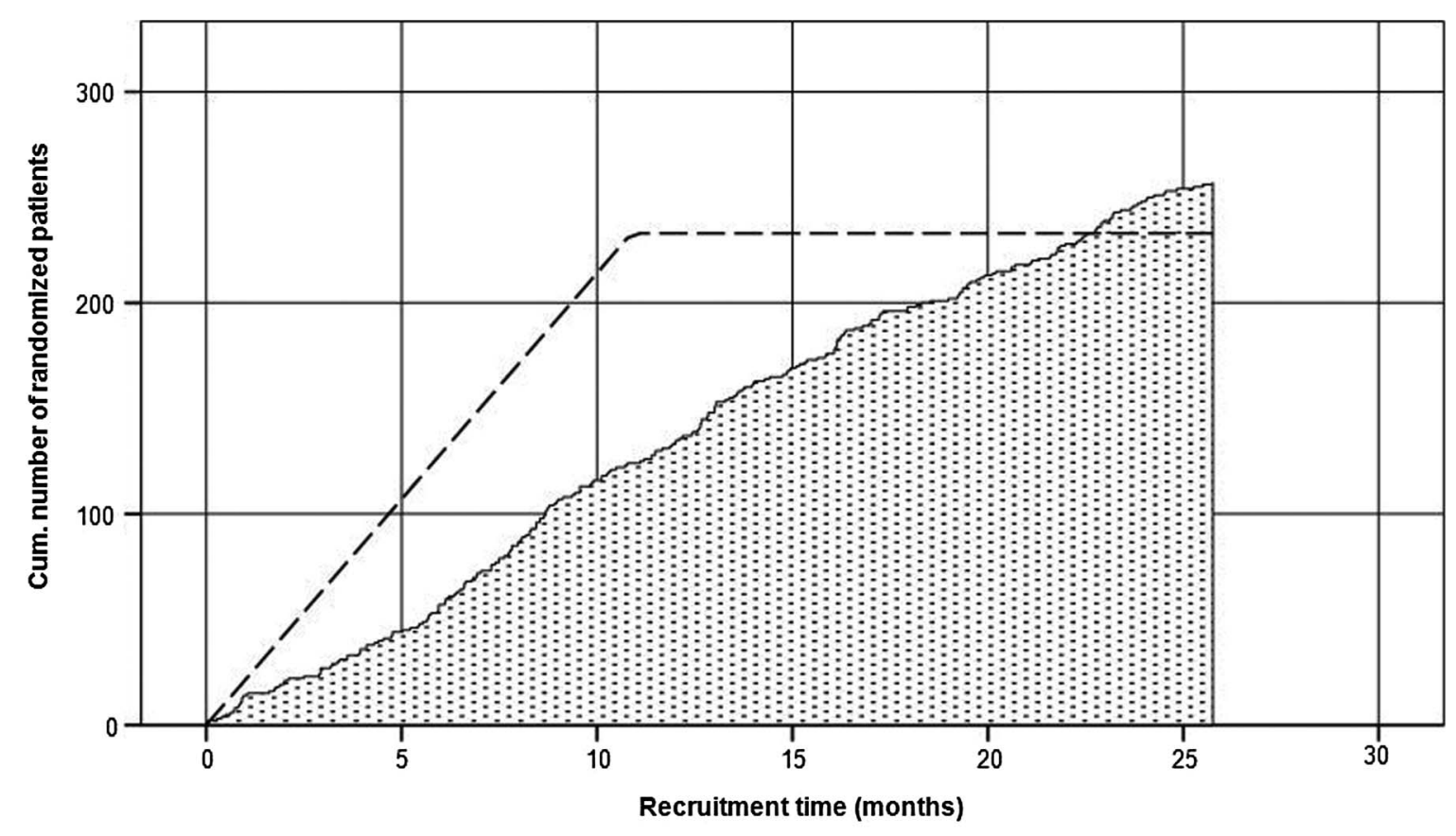

Area: Cum. number of randomized patients Dashed line: Cum. number of expected patients

Figure 2 Enrolment of patients over time. 


\section{Trial status}

Overall, 14 centres and 34 surgeons participated in the study, 18 surgeons enrolled only in arms E1 and C with allocation ratio 1:1 and 16 surgeons enrolled in all three arms, including $23 / 25 \mathrm{G}$ vitrectomy with gas (E2) with ratio 1:1:1. During the enrolment phase from June 2011 to August 2013, a total of 257 patients with PRD were included (see figure 2), 100 patients were randomly assigned to arm E1, 57 patients to arm E2 and 100 patients to arm C, respectively. Because the surgeons who were proficient in all three techniques tended to recruit more patients than expected, that is, eventually 57 instead of 33 patients as given in the protocol, the local ethics committees were timely asked for reapproval which they granted.

\section{SUMMARY AND CONCLUSION}

The additional implantation of a scleral buckle in the treatment of retinal detachments with vitrectomy implies an additional trauma. The $360^{\circ}$ incision of the conjunctiva and exposition of the sclera lead to consecutive scarring and surface alterations and the impact of the buckle on the shape of the eye results in a significant myopisation. The VIPER study will demonstrate whether the additional risks of a circumferential scleral buckle combined with vitrectomy are justified by a superior success rate. Moreover, in view of the increasing use of transconjunctival vitrectomy to treat retinal detachments, it seems mandatory to investigate whether patients benefit from an additional buckle before this technique is, possibly prematurely, abandoned due to technical progress.

\section{Trial organisation}

Coordinating investigator is Peter Walter, Department of Ophthalmology, University of Aachen (RWTH). Project coordination, correspondence with regulatory authorities, data management and central monitoring were performed by the Clinical Trials Centre Cologne (ZKS Köln), University of Cologne. Statistical design, randomisation and data analysis were performed by the Institute of Medical Statistics, Informatics and Epidemiology (IMSIE), University of Cologne. Trial procedures were implemented according to the standard operating procedures of ZKS Köln and IMSIE.

Collaborators VIPER Study Group Surgeons, in alphabetical order: Agostini H (Freiburg), Aisenbrey S (Tübingen), Bartz-Schmidt U (Tübingen), Bornfeld N (Essen), Clemens C (Münster), Dahlke C (Cologne), Eter N (Münster), Fauser S (Cologne), Feltgen N (Göttingen), Gamulescu MA (Regensburg), Gök M (Essen), Helbig H (Regensburg), Hillenkamp J (Kiel), Holz F (Bonn), Jochmann C (Leipzig), Joussen A (Berlin), Junker B (Freiburg), Kirchhof B (Cologne), Lappas A (Cologne), Lommatzsch A (Münster), Lüke M (Lübeck), Mazinani B (Aachen), Meier P (Leipzig), Neß T (Freiburg), Nestler A (Leipzig), Partsch M (Tübingen), Pielen A (Freiburg), Rasche W (Leipzig), Ritzau-Tondrow U (Göttingen), Rössler G (Aachen), Roider J (Kiel), Rudolf M (Lübeck), Uhlig C (Münster), Walter P (Aachen), Wiedemann P (Leipzig). Data management, central monitoring: Pfeiffer A (Cologne). Medical statistics: Hellmich M (Cologne), Schiller P (Cologne). Project management: Limburg E (Cologne), Weiß C (Cologne).

Contributors The following persons have contributed to the planning, conduct and reporting of the study: BM, SB, PS, HA, HH, EL, MH and PW.

Funding The study was supported by retina.net, the German Competence Network for Clinical Studies in Vitreoretinal Surgery. retina.net received grants from the Jackstädt Foundation, the German Retina Society and the German Ophthalmological
Society (DOG). Moreover, ZKS Köln was supported by BMBF grant 01KN1106. These funders had no participation in or authority on study design, data or writing of the report.

Competing interests None declared.

\section{Patient consent Obtained.}

Ethics approval The local ethics committees of all participating institutions.

Provenance and peer review Not commissioned; externally peer reviewed.

Open Access This is an Open Access article distributed in accordance with the Creative Commons Attribution Non Commercial (CC BY-NC 4.0) license, which permits others to distribute, remix, adapt, build upon this work non-commercially, and license their derivative works on different terms, provided the original work is properly cited and the use is non-commercial. See: http://creativecommons.org/ licenses/by-nc/4.0/

\section{REFERENCES}

1 Ogasawara H, Feke GT, Yoshida A, et al. Retinal blood flow alterations associated with scleral buckling and encircling procedures. Br J Ophthalmol 1992;76:275-9.

2 Devenyi RG, de Carvalho Nakamura H. Combined scleral buckle and pars plana vitrectomy as a primary procedure for pseudophakic retinal detachments. Ophthalmic Surg Lasers 1999;30:615-8.

3 Desai UR, Strassman IB. Combined pars plana vitrectomy and scleral buckling for pseudophakic and aphakic retinal detachments in which a break is not seen preoperatively. Ophthalmic Surg Lasers 1997;28:718-22.

4 Pournaras CJ, Kapetanios AD. Primary vitrectomy for pseudophakic retinal detachment: a prospective non-randomized study. Eur J Ophthalmol 2003;13:298-306.

5 Wickham L, Connor M, Aylward GW. Vitrectomy and gas for inferior break retinal detachments: are the results comparable to vitrectomy, gas, and scleral buckle? Br J Ophthalmol 2004;88:1376-9.

6 Stangos AN, Petropoulos IK, Brozou CG, et al. Pars-plana vitrectomy alone vs vitrectomy with scleral buckling for primary rhegmatogenous pseudophakic retinal detachment. Am J Ophthalmol 2004;138:952-8.

7 Adelman RA, Parnes AJ, Ducournau D, et al. Strategy for the management of uncomplicated retinal detachments: the European vitreo-retinal society retinal detachment study report 1. Ophthalmology 2013;120:1804-8.

8 Heimann $\mathrm{H}$, Bartz-Schmidt KU, Bornfeld N, et al. Scleral buckling versus primary vitrectomy in rhegmatogenous retinal detachment: a prospective randomized multicenter clinical study. Ophthalmology 2007;114:2142-54.

9 Dell'Omo R, Barca F, Tan HS, et al. Pars plana vitrectomy for the repair of primary, inferior rhegmatogenous retinal detachment associated to inferior breaks. A comparison of a 25-gauge versus a 20-gauge system. Graefes Arch Clin Exp Ophthalmol 2013;251:485-90.

10 Lewis SA, Miller DM, Riemann CD, et al. Comparison of 20-, 23-, and 25-gauge pars plana vitrectomy in pseudophakic rhegmatogenous retinal detachment repair. Ophthalmic Surg Lasers Imaging 2011;42:107-13.

11 Hikichi T, Kosaka S, Takami K, et al. Surgical outcomes of 23- and 20-gauge vitrectomies for rhegmatogenous retinal detachment associated with posterior vitreous detachment. Ophthalmic Surg Lasers Imaging 2011;42:376-82.

12 Albrieux M, Rouberol F, Bernheim D, et al. Comparative study of 23-gauge vitrectomy versus 20-gauge vitrectomy for the treatment of rhegmatogenous retinal detachment. Graefes Arch Clin Exp Ophthalmol 2011;249:1459-68.

13 Von Fricken MA, Kunjukunju N, Weber C, et al. 25-Gauge sutureless vitrectomy versus 20-gauge vitrectomy for the repair of primary rhegmatogenous retinal detachment. Retina 2009;29:444-50.

14 Dupont WD, Plummer WD Jr. Power and sample size calculations. A review and computer program. Controlled Clinical Trials 1990;11:116-28.

15 Donner A. Approaches to sample size estimation in the design of clinical trials-a review. Stat Med 1984;3:199-214.

16 Committee for Proprietary Medicinal P. Points to consider on switching between superiority and non-inferiority. Br J Clin Pharmacol 2001;52:223-8.

17 Mantel N, Haenszel W. Statistical aspects of the analysis of data from retrospective studies of disease. J Nat/ Cancer Inst 1959;22:719-48.

18 Aaa A. Uniform requirements for manuscripts submitted to biomedical journals. International Committee of Medical Journal Editors. Ann Intern Med 1982; 96(6 Pt 1):766-71. 